\title{
On the Napsuciale-Kirchbach Formalism for Spin 3/2 Field Theory
}

\author{
Haryanto M. Siahaan and Triyanta \\ Theoretical Energy Physics Laboratory, \\ Theoretical High Energy Physics and Instrumentation Research Division \\ Faculty of Mathematics and Natural Sciences \\ Institut Teknologi Bandung, Bandung \\ e-mail: triyanta@fi.itb.ac.id
}

\begin{abstract}
We consider the newly approach by Napsuciale and Kirchbach as an alternative formalism for spin-3/2 fields. The Feynman rules for interacting case are derived. Gauge invariance property in this formalism is shown from the corresponding invariant Compton scattering amplitude related to gauge transformation of the polarization vector.
\end{abstract}

Keywords: Spin-3/2 field, Compton scattering amplitude, Gauge invariance

\section{Introduction}

Finding a consistent theory for quantum fields with spin $3 / 2$ has been a classic problem in theoretical physics. Standard formulation for spin 3/2 particles was proposed by W. Rarita and J. Schwinger in $1941^{1)}$. But this formalism has some issues related to the Lorentz invariance property in the presence of strong magnetic fields (Velo-Zwanziger problem) and extra degrees of freedom for describing spin $3 / 2$ particle wave functions ${ }^{2)}$. A new approach for describing particles with spin $3 / 2$ proposed by Napsuciale and Kirchbach ${ }^{3-6)}$ attracts many physicists, including us, to pay attention on spin 3/2 particles.

Although, there are still no single particles with spin 3/2 observed in laboratories there exist baryon resonances with spins ranging from $3 / 2$ to $9 / 2$. Efforts to describe this resonance also have become one of active researches in theoretical physics ${ }^{7,8)}$.

In this paper, we analyze the gauge invariance property of the Compton scattering amplitude of the spin $3 / 2$ fields in the Napsuciale-Kirchbach (NK) formalism.

\section{The NK Formalism}

As described by a series of their papers ${ }^{3-5)}$, Napsuciale and Kirchbach construct a new equation of motion for fields with spin based on eigen equation of squared Pauli-Lubanski operator $W^{\alpha}$ :

$$
\begin{aligned}
& W^{2} \Psi^{(m, s)}=-p^{2}(s(s+1)) \Psi^{(m, s)}, \\
& \left(W^{2}\right)_{a b \alpha \beta}=-\left\{2\left(g_{\alpha \beta} g_{\mu \nu}-g_{\alpha \nu} g_{\beta \mu}\right) \delta_{a b}+\right. \\
& \frac{1}{4} g_{\alpha \beta}\left(\sigma_{\rho \mu} \sigma_{\nu}^{\rho}\right)_{a b}+ \\
& \left.\frac{1}{2}\left(\varepsilon_{\alpha \beta \mu}^{\lambda} \gamma^{5} \sigma_{\lambda \nu} \varepsilon_{\alpha \beta \nu}^{\lambda} \gamma^{5} \sigma_{\lambda \mu}\right)_{a b}\right\} p^{\mu} p^{v}
\end{aligned} .
$$

The fields $\Psi^{(m, s)}$ denote eigenfunctions for specific mass $m$ and spin $s$, and $p$ is the momentum. We restrict our discussion for fields that have spin's degree of freedom in $s$ and $(s-1)$. The main prescription in this formalism is to construct the equation of motion (e.o.m.) for each $\Psi$ which satisfies the following conditions:

$$
\begin{aligned}
& {\left[\hat{P}^{(m, s)}(p)\right]^{2}=\hat{P}^{(m, s)}(p),} \\
& \hat{P}^{(m, s)}(p) \hat{P}^{(m, s-1)}(p)=0, \\
& {\left[\hat{P}^{(m, s-1)}(p)\right]^{2}=\hat{P}^{(m, s-1)}(p),} \\
& \hat{P}^{(m, s)}(p)+\hat{P}^{(m, s-1)}(p)=1 .
\end{aligned}
$$

So we can write the e.o.m. as

$$
\hat{P}^{(m, s)}(p) \Psi^{(m, s)}=\Psi^{(m, s)},
$$

with constraint

$$
\hat{P}^{(m, s-1)}(p) \Psi^{(m, s)}=0,
$$

and mass shell condition

$$
\left(p^{2}-m^{2}\right) \Psi^{(m, s)}=0 .
$$

For spin 3/2 system, we can write the e.o.m as

$$
\left[-\frac{1}{3} T_{A B \mu \nu} p^{\mu} p^{\nu}-\left(\frac{1}{4} p^{2}+m^{2}\right) \delta_{A B}\right] \psi^{B}=0,
$$

where $\psi^{B}$ is spin $3 / 2$ field in vector spinor representation, and related to (1), $\left(W^{2}\right)_{A B} \equiv T_{A B \mu \nu} p^{\mu} p^{\nu}$. We have used index notation where $\{A, B\}$ could be Lorentz indices $\{\alpha, \beta\}$, or spinor indices $\{a, b\}$. In another simple form, (6) can be written as

$$
\left[-\Gamma_{A B \mu \nu} p^{\mu} p^{\nu}+m^{2} \delta_{A B}\right] \psi^{B}=0,
$$

with

$$
\Gamma_{A B \mu \nu}=-\frac{1}{3}\left(T_{A B \mu \nu}+\frac{3}{4} \delta_{A B} g_{\mu \nu}\right) .
$$

We will use the expressions (7) and (8) for the next parts of this paper.

\section{Spin 3/2 Lagrangian and Feynman Rules in NK Formalism}

The free Lagrangian for the spin 3/2 field $\psi^{\beta}$ fulfilling the eq. (7) can be written as 


$$
\mathcal{L}_{\text {free }}=-\left(\partial^{\mu} \bar{\psi}^{\alpha}\right) \Gamma_{\alpha \beta \mu \nu} \partial^{\nu} \psi^{\beta}+m^{2} \bar{\psi}^{\alpha} \psi_{\alpha},
$$

with related Noether current

$$
j_{\mu}=\left(i \partial^{\nu} \bar{\psi}^{\alpha}\right) \Gamma_{\alpha \beta \nu \mu} \psi^{\beta}-\bar{\psi}^{\alpha} \Gamma_{\alpha \beta \mu \nu}\left(i \partial^{\nu} \psi^{\beta}\right) .
$$

This current satisfies continuity equation, $\partial^{\mu} j_{\mu}=0$. Interaction with external electromagnetic field will be described by replacing Lagrangian (9) by

$$
\mathcal{L}=-\left(D^{\dagger \mu} \bar{\psi}^{\alpha}\right) \Gamma_{\alpha \beta \mu \nu} D^{\nu} \psi^{\beta}+m^{2} \bar{\psi}^{\alpha} \psi_{\alpha}
$$

where $D^{\mu}=\partial^{\mu}-i e A^{\mu}$ is the covariant derivative (with particle charge $-e$ ). Writting $\mathcal{L}=\mathcal{L}_{\text {free }}+\mathcal{L}_{\text {int }}$ the interaction part of Lagrangian can be read as

$$
\mathcal{L}_{\text {int }}=e j_{\mu} A^{\mu}+e^{2} \bar{\psi}^{\alpha} \Gamma_{\alpha \beta \nu \mu} \psi^{\beta} A^{\mu} A^{\nu} .
$$

Let us now consider the Feynman rules of the theory. The propagator for spin 3/2 fields can be obtained as

$$
\Pi_{\alpha \beta}=\frac{\Delta_{\alpha \beta}}{\left(p^{2}-m^{2}+i \varepsilon\right)},
$$

where

$$
\begin{gathered}
\Delta_{\alpha \beta}=-g_{\alpha \beta}+\frac{2}{3 m^{2}} p_{\beta} p_{\alpha}+ \\
\frac{1}{3 m^{2}}\left(p_{\alpha} \gamma_{\beta}+p_{\beta} \gamma_{\alpha}\right) \not p+ \\
-\frac{1}{3 m^{2}} \gamma_{\alpha} \not p \gamma_{\beta} \not p . \\
\frac{\beta}{P} \\
:-i \Pi_{\alpha \beta}
\end{gathered}
$$

We have two types of vertices, namely, the $\bar{\psi} A \psi$ and $\bar{\psi} A^{2} \psi$ vertices and are depicted as the following.

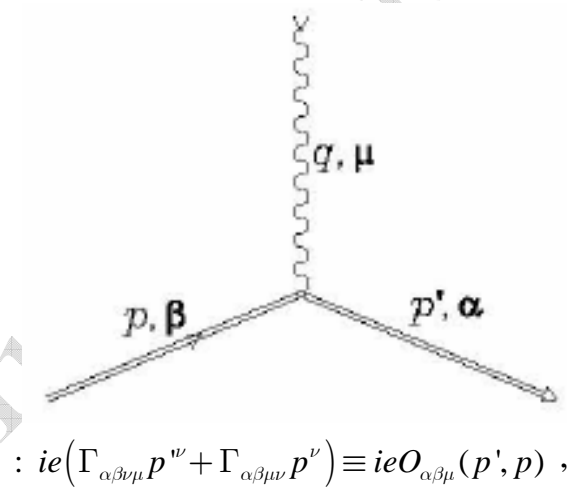

(16a)

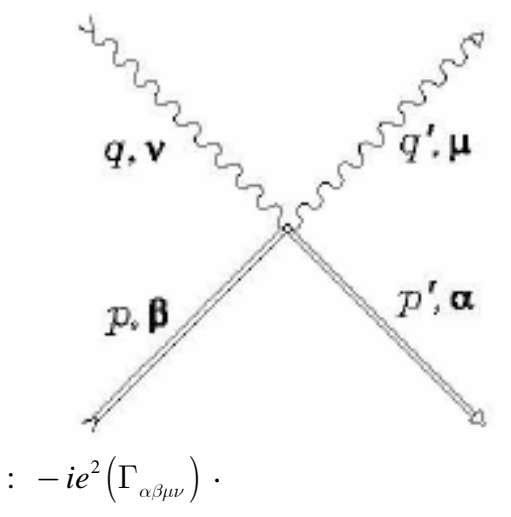

\section{Compton Scattering Amplitude and Gauge Invariance}

We consider the lowest order of Feynman diagram for the Compton scattering. Based on the Feynman rules the lowest order of the Compton scattering can be depicted as the following:

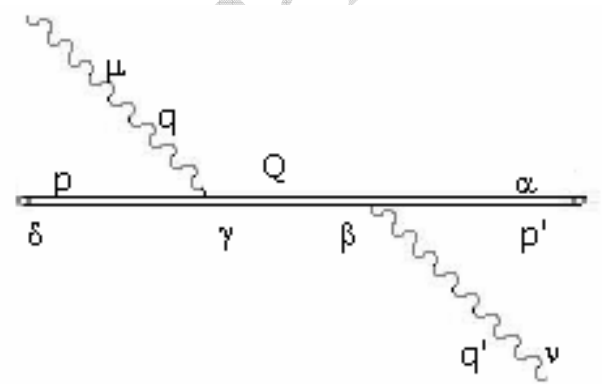

(a)

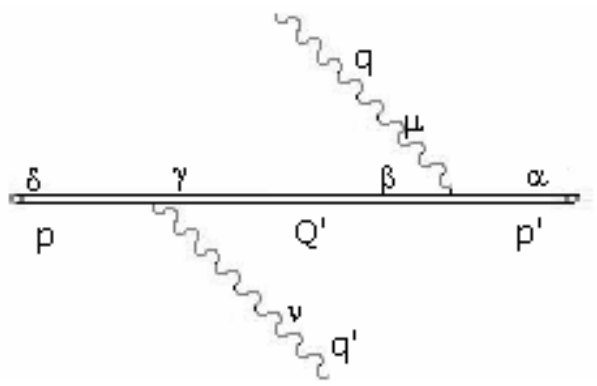

(b)

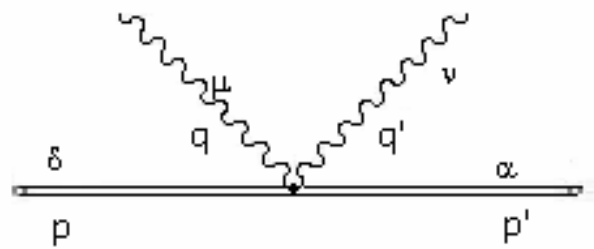

(c)

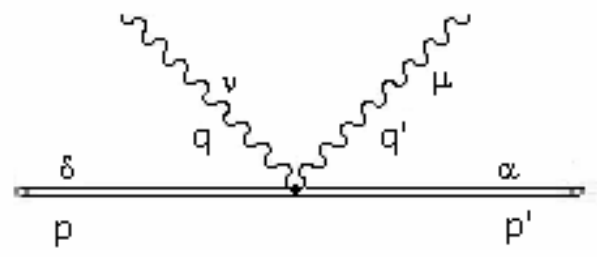

(d)

In the diagrams above, time's direction is considered from left to right. 
Accordingly, our total Compton amplitude can be written as:

$$
\begin{aligned}
M & =e^{2} \bar{u}^{\alpha}\left(p^{\prime}\right)\left\{O_{\alpha \beta \nu}\left(p^{\prime}, Q\right)\left(\Pi^{\beta \gamma}(Q)\right)\right. \\
& \times O_{\gamma \delta \mu}(Q, p)+O_{\alpha \beta \mu}\left(p^{\prime}, Q^{\prime}\right) \\
& \times\left(\Pi^{\beta \gamma}\left(Q^{\prime}\right)\right) O_{\gamma \delta \nu}\left(Q^{\prime}, p\right) \\
& \left.-\left(\Gamma_{\alpha \delta \nu \mu}+\Gamma_{\alpha \delta \mu \nu}\right)\right\} u^{\delta}(p) \varepsilon^{\nu} *\left(q^{\prime}\right) \varepsilon^{\mu}(q) .
\end{aligned}
$$

To test the gauge invariance property of (17), we can perform shifting the photon polarization in amplitude,

$$
\begin{aligned}
& \varepsilon^{\nu *}\left(q^{\prime}\right) \rightarrow \varepsilon^{\nu *}\left(q^{\prime}\right)+q^{\nu} \theta^{\prime *}, \\
& \varepsilon^{\mu}(q) \rightarrow \varepsilon^{\mu}(q)+q^{\mu} \theta .
\end{aligned}
$$

The $\theta$ expression in (18) is arbitrary complex scalar functions.

We will show that this shifting will give our amplitude to be invariant. The difference of Compton amplitude (17) between before and after shifting can be written as the following.

$$
\begin{aligned}
& M^{\prime}-M=e^{2} \bar{u}^{\alpha}\left(p^{\prime}\right)\left\{O_{\alpha \beta \nu}\left(p^{\prime}, Q\right)\left(\Pi^{\beta \gamma}(Q)\right)\right. \\
& \times O_{\gamma \delta \mu}(Q, p)+O_{\alpha \beta \mu}\left(p^{\prime}, Q^{\prime}\right) \\
& \times\left(\Pi^{\beta \gamma}\left(Q^{\prime}\right)\right) O_{\gamma \delta \nu}\left(Q^{\prime}, p\right) \\
& \left.-\left(\Gamma_{\alpha \delta \nu \mu}+\Gamma_{\alpha \delta \mu \nu}\right)\right\} u^{\delta}(p) \\
& \times\left\{\varepsilon^{\nu *}\left(q^{\prime}\right) q^{\mu} \theta+q^{\nu} \theta^{\prime *} \varepsilon^{\mu}(q)+q^{\nu} \theta^{\prime *} q^{\mu} \theta\right\}
\end{aligned}
$$

By showing that $M^{\prime}-M=0$, it proves gauge invariance property of the theory. Using the fact that $T_{\nu} q^{\nu}=0$ (in the same fashion with ref. [9], but different Feynman rules for 16.b) for $M=T_{\nu} \varepsilon^{\nu}$ (see the appendix), the coefficient of $\theta, \theta^{*}$, and $\theta \theta^{*}$ in (19) would vanish, as we need.

\section{Conclusions and Discussions}

We have performed gauge invariance test for lowest order Compton Scattering in the NK formalism. Since this theory also free from Velo Zwanziger problem ${ }^{5}$, it seems that this theory could replace the Rarita Scwhinger description of spin 3/2 particles. This conclusion perhaps is rather rough. It would be interesting to consider higher order, such as the one-loop diagrams of the Compton scattering. One could also calculate the pion nucleon scattering that includes $\Delta(1232)$ resonance using this formalism. These will be our next considerations.

\section{Acknowledgement}

The authors thanks to Prof. Napsuciale for useful discussion.

\section{References}

1. W. Rarita, and J. Schwinger, On the Theory of Particles with Half-Integral Spin, Phys. Rev., 60, 6, 1941.
2. G. Velo, and D. Zwanziger, Propagation and Quantization of Rarita-Schwinger waves in an External Electromagnetic Potential, Phys. Rev., 186, 1337, 1996.

3. M. Napsuciale, and M. Kirchbach, Avoiding Superluminal Propagation of Higher Spin Waves ia Projectors onto $\mathrm{W}^{2}$ Invariant Subspaces, J.Math.Phys., 45, 4515-4523, 2004.

4. M. Napsuciale, and M. Kirchbach, High Spins Beyond Rarita-Schwinger Framework, hepph/0407179.

5. M. Napsuciale and M. Kirchbach, Spin 3/2 Beyond Rarita-Schwinger Framework, Eur. Phys. J., A29, 289-306, 2006.

6. L.M. Rico, and M. Kirchbach, Causal Propagation of Spin-Cascades, hep-ph/0707006.

7. M. Napsuciale, and J.L. Lucio M., Spin 3/2 Interacting Fields and Heavy Baryon Chiral Perturbation Theory, hep-ph/9605262.

8. V. Pascalutsa and D. R. Phillips, Effective Theory of the Delta (1232) in Compton Scattering off the Nucleon, Phys.Rev. C., 67, 055202, 2003.

9. M. Napsuciale, private communication.

\section{Appendix}

The Compton amplitude for each diagram (a, b, c, and d) can be decomposed into ${ }^{9)}$ :

$$
\begin{aligned}
M & =M_{a}+M_{b}+M_{c}+M_{d} \\
& =\left(\mathrm{M}^{(a)}{ }_{\nu}+\mathrm{M}^{(b)}{ }_{\nu}+\mathrm{M}^{(c)}{ }_{\nu}+\mathrm{M}^{(c)}{ }_{\nu}\right) \varepsilon^{\nu}
\end{aligned}
$$

Related to diagram (a), we can perform the following calculation:

$$
\begin{aligned}
\mathrm{M}^{(a)}{ }_{\nu} q^{\nu}{ }^{\prime}=-e^{2} \bar{u}^{\alpha}\left(p^{\prime}\right)\left\{O_{\alpha \beta \nu}\left(p^{\prime}, Q\right)\left(\Pi^{\beta \gamma}(Q)\right)\right. \\
\left.\quad \times O_{\gamma \delta \mu}(Q, p)\right\} u^{\delta}(p) q^{\nu} \varepsilon^{\mu}(q) \\
=-e^{2} \bar{u}^{\alpha}\left(p^{\prime}\right)\left\{\left\{K_{\alpha \beta}\left(p^{\prime}\right)-K_{\alpha \beta}(Q)\right\}\left(\Pi^{\beta \gamma}(Q)\right)\right. \\
\left.\quad \times O_{\gamma \delta \mu}(Q, p)\right\} u^{\delta}(p) \varepsilon^{\mu}(q) \\
\left.=-e^{2} \bar{u}^{\alpha}\left(p^{\prime}\right) O_{\alpha \delta \mu}(Q, p)\right\} u^{\delta}(p) \varepsilon^{\mu}(q)
\end{aligned}
$$

For calculation (A2), we have used these relations:

$$
\begin{aligned}
O_{\alpha \beta \nu}\left(p^{\prime}, Q\right) q^{\nu} ' & =O_{\alpha \beta \nu}\left(p^{\prime}, Q\right)\left\{Q^{\nu}-p^{\nu}\right\} \\
& =K_{\alpha \beta}\left(p^{\prime}\right)-K_{\alpha \beta}(Q), \\
K_{\alpha \beta}(Q) \Pi^{\beta \gamma}(Q) & =-g_{\alpha}^{\gamma}+m^{2} \Pi_{\alpha}^{\gamma}(Q), \\
\bar{u}^{\alpha}\left(p^{\prime}\right) K_{\alpha \beta}\left(p^{\prime}\right) & =m^{2} \bar{u}_{\beta}\left(p^{\prime}\right),
\end{aligned}
$$

and $q^{\prime}=Q-p^{\prime}$.

The similar method also can be used to analyze diagram (b).

$$
\begin{aligned}
& L_{\nu} q^{\prime \nu}=-e^{2} \bar{u}^{\alpha}\left(p^{\prime}\right)\left\{O_{\alpha \beta \mu}\left(p^{\prime}, Q^{\prime}\right)\left(\Pi^{\beta \gamma}\left(Q^{\prime}\right)\right)\right. \\
& \left.\times O_{\gamma \delta \nu}\left(Q^{\prime}, p\right)\right\} u^{\delta}(p) q^{\prime \nu} \varepsilon^{\mu}(q) \\
& =-e^{2} \bar{u}^{\alpha}\left(p^{\prime}\right)\left\{O_{\alpha \beta \mu}\left(p^{\prime}, Q^{\prime}\right)\left(\Pi^{\beta \gamma}\left(Q^{\prime}\right)\right)\right.
\end{aligned}
$$




$$
\begin{aligned}
& \left.\times\left\{K_{\gamma \delta}\left(Q^{\prime}\right)-K_{\gamma \delta}(p)\right\}\right\} u^{\delta}(p) \varepsilon^{\mu}(q) \\
& =+e^{2} \bar{u}^{\alpha}\left(p^{\prime}\right)\left\{O_{\alpha \delta \mu}\left(p^{\prime}, Q^{\prime}\right) u^{\delta}(p) \varepsilon^{\mu}(q)\right.
\end{aligned}
$$

We have used the relations $q^{\prime}=p-Q^{\prime}$ and $O_{\gamma \delta \nu}\left(Q^{\prime}, p\right) q^{\nu}{ }^{\prime}=K_{\gamma \delta}\left(Q^{\prime}\right)-K_{\gamma \delta}(p)$ for (A5).

Then for the second order diagram (c) and (d), we can compute as the following.

$$
\begin{aligned}
& \left(M_{\nu}+N_{\nu}\right) q^{\prime \nu}=-e^{2} \bar{u}^{\alpha}\left(p^{\prime}\right)\left\{\Gamma_{\alpha \delta \nu \mu}+\Gamma_{\alpha \delta \mu \nu}\right\} \\
& \times u^{\delta}(p) q^{\nu} \varepsilon^{\mu}(q)=-e^{2} \bar{u}^{\alpha}\left(p^{\prime}\right)\left\{\Gamma_{\alpha \delta \nu \mu}\left(Q^{\nu}-p^{\nu}\right)\right. \\
& \left.+\Gamma_{\alpha \delta \mu \nu}\left(p^{\nu}-Q^{\nu}\right)\right\} u^{\delta}(p) \varepsilon^{\mu}(q)
\end{aligned}
$$

$$
\begin{aligned}
& =e^{2} \bar{u}^{\alpha}\left(p^{\prime}\right) O_{\alpha \delta \mu}(Q, p) u^{\delta}(p) \varepsilon^{\mu}(q) \\
& -e^{2} \bar{u}^{\alpha}\left(p^{\prime}\right) O_{\alpha \delta \mu}\left(p^{\prime}, Q^{\prime}\right) u^{\delta}(p) \varepsilon^{\mu}(q)
\end{aligned}
$$

where we have used the relation

$$
\left(\Gamma_{\alpha \beta \nu \mu} p^{\prime \prime}+\Gamma_{\alpha \beta \mu \nu} p^{\nu}\right) \equiv-O_{\alpha \beta \mu}\left(p^{\prime}, p\right) \cdot
$$

From the results of (A2), (A5), and (A6), we can write that

$$
\left(K_{\mu}+L_{\mu}+M_{\mu}+N_{\mu}\right) q^{\mu} \equiv T_{\mu} q^{\mu}=0 .
$$

Also for coefficient of $\theta, \theta^{*}$, and $\theta \theta^{*}$ in (19) can be proved to be vanishing in the similar way. 